lymphoid tissues, not to induce autophagy (reviewed in ref. 17). Indeed, Espert et al. noted that SDF-1 was unable to trigger autophagy in their assays (4), indicating that the binding of HIV envelope glycoproteins to CXCR4 induces distinct intracellular signaling events compared with the natural ligand (Figure 1). These findings raise the possibility that viral proteins may selectively transduce signals through chemokine receptors that redirect the target cell away from its normal function and toward a cell suicide program driven by autophagy activation.

\section{Conclusion}

Espert et al. (4) report a very intriguing observation, namely that the HIV envelope glycoprotein induces CXCR4dependent autophagy of uninfected lymphocytes, which is required for both caspase-dependent, apoptotic cell death and caspase-independent, nonapoptotic cell death. This work eloquently establishes that autophagy can function upstream of apoptosis in cell death signaling, that a viral envelope glycoprotein can trigger autophagy-dependent bystander cell death by binding to a cell surface receptor, and that a chemokine receptor can activate an autophagy-dependent death program in response to engagement by a viral protein. Future studies will be needed to determine whether the HIV envelope glycoprotein mediates autophagy-dependent cell death in bystander CXCR4-expressing $\mathrm{CD}^{+}{ }^{+} \mathrm{lym}$ phocytes in HIV-infected patients and the relative contribution of this process to the progressive decline in numbers of $\mathrm{CD}^{+} \mathrm{T}$ cells in patients with AIDS.

Address correspondence to: Beth Levine, University of Texas Southwestern Medical Center, 5323 Harry Hines Boulevard, Dallas, Texas 75390-9113, USA. Phone: (214) 648-2202; Fax: (214) 648-0284; E-mail: beth.levine@utsouthwestern.edu.

1. Alimonti, J.B., Ball, B., and Fowke, K.R. 2003. Mechanisms of CD4+ T lymphocyte cell death in human immunodeficiency virus infection and AIDS. J. Gen. Virol. 84:1649-1661.

2. Ahr, B., Robert-Hebmann, V., Devaux, C., and BairdPiechaczyk, M. 2004. Apoptosis of uninfected cells induced by HIV envelope glycoproteins. Retrovirology. 1:1-12.

3. Bell, D.J., and Dockrell, D.H. 2003. Apoptosis in HIV-1 infection. J. Eur. Acad. Dermatol. Venereol. 17:178-183.

4. Espert, L., et al. 2006. Autophagy is involved in T cell death after binding of HIV-1 envelope proteins to CXCR4. J. Clin. Invest. 116:2161-2172. doi:10.1172/JCI26185.
5. Levine, B., and Klionsky, D.J. 2004. Development by self-digestion: molecular mechanisms and biological functions of autophagy. Dev. Cell. 6:463-477.

6. Komatsu, M., et al. 2006. Loss of autophagy in the central nervous system causes neurodegeneration in mice. Nature. 441:880-884.

7. Hara, T., et al. 2006. Suppression of basal autophagy in neural cells causes neurodegenerative disease in mice. Nature. 441:885-889.

8. Levine, B., and Yuan, J. 2005. Autophagy in cell death: an innocent convict? J. Clin. Invest. 115:2679-2688. doi:10.1172/JCI26390.

9. Boya, P., et al. 2005. Inhibition of macroautophagy triggers apoptosis. Mol. Cell. Biol. 25:1025-1040.

10. Xue, L., Fletcher, G.C., and Tolkovsky, A.M. 1999. Autophagy is activated by apoptotic signalling in sympathetic neurons: an alternative mechanism of death execution. Mol. Cell. Neurosci. 14:180-198.

11. Xue, L., Borutaite, V., and Tolkovsky, A.M. 2002. Inhibition of mitochondrial permeability transition and release of cytochrome c by anti-apoptotic nucleoside analogues. Biochem. Pharmacol. 64:441-449.

12. Levine, B. 2006. Autophagy in antiviral host defense. Wiley-VCH. Weinheim, Germany. 227-241.

13. Liu, Y., et al. 2005. Autophagy regulates programmed cell death during the plant innate immune response. Cell. 121:567-577.

14. Wileman, T. 2006. Aggresomes and autophagy generate sites for virus replication. Science. 312:875-878.

15. Scarlatti, G., et al. 1997. In vivo evolution of HIV-1 co-receptor usage and sensitivity to chemokinemediated suppression. Nat. Med. 3:1259-1265.

16. Philpott, S.M. 2003. HIV-1 coreceptor usage, transmission, and disease progression. Curr. HIV Res. 1:217-227.

17. Kucia, M., et al. 2005. Stromal cell-derived factor1alpha/CXCL12-induced. J. Mol. Histol. 35:233-245.

\title{
Costimulation couture: a designer approach to regulating autoimmunity
}

\author{
Mohammed Javeed Ansari and Mohamed H. Sayegh
}

\begin{abstract}
Transplantation Research Center, Renal Division, Brigham and Women's Hospital and Children's Hospital Boston,
\end{abstract} Harvard Medical School, Boston, Massachusetts, USA.

\begin{abstract}
Negative or inhibitory costimulatory pathways regulate $T$ cell activation and play a role in peripheral tolerance. Targeting these pathways harnesses the physiologic mechanisms of regulating autoimmunity and could prove beneficial for the therapy of autoimmune diseases. However, attempts at targeting these pathways have been fraught with difficulties. In this issue of the JCI, Fife et al. describe a creative approach for targeting CTL-associated antigen 4 (CTLA-4) on activated $T$ cells via genetically engineered $B$ cells to prevent autoimmune diabetes in the NOD mouse (see the related article beginning on page 2252). Novel "designer" strategies targeting negative costimulatory pathways provide reasons for optimism in the search for a cure for devastating autoimmune diseases.
\end{abstract}

Nonstandard abbreviations used: CTLA-4, CTL-associated antigen 4; PD-1, programmed death-1; PD-L1, PD-1 ligand; scFv, single-chain, membranebound anti-CTLA-4 antibody.

Conflict of interest: The authors have declared that no conflict of interest exists.

Citation for this article: J. Clin. Invest. 116:2080-2083 (2006). doi:10.1172/JCI29455.

\section{Evolution of the concept of costimulation: positive and negative costimulatory signals}

In the early 1970s, Bretscher and Cohn proposed the 2-signal model for lymphocyte, specifically B cell, activation (1). Lafferty and colleagues later extended this model to $T$ cell activation $(2,3)$. Realization that efficient $\mathrm{T}$ cell activation requires 2 signals (first, signal 1, an antigen-specific signal mediated via the TCR; second, signal 2, a noncognate costimulatory signal) led to the search for the costimulatory signal and identification of the CD28-B7 pathway in the early 1990 s $(4,5)$.

Soon after the discovery of the CD28-B7 positive costimulatory pathway, it became apparent that CTL-associated antigen 4 (CTLA-4), a second inducible receptor that is homologous to $\mathrm{CD} 28$ and binds with higher affinity to B7-1 and B7-2, could function as a negative regulator of $\mathrm{T}$ cell activation (6, 7). CTLA-4 is also constitutively expressed on Tregs (8) and is important for their function (9-11) and generation $(12,13)$ while CD28 signaling is critical for Treg homeo- 
stasis (14). In addition, CTLA-4-mediated negative signaling is required for establishing peripheral tolerance (15-19).

Over the past 15 years, studies have focused on targeting the CD28-B7 pathway to induce tolerance in autoimmunity (20) and transplantation (21). Recently, the fusion protein CTLA-4Ig, which binds B7-1 and B7-2 and blocks CD28-B7 costimulation, has been approved for the treatment of rheumatoid arthritis (22), and it is currently being tested in several other human autoimmune diseases. The second-generation fusion protein LEA29Y is in clinical development for organ transplantation (23). However, indiscriminate blockade of both CD28 and CTLA-4 raises the potential concern that this strategy may interfere with tolerance induction and restrict the therapeutic potential of CTLA-4Ig (14). Admittedly, selective targeting of CTLA-4 and other negative $T$ cell costimulatory pathways to induce tolerance in vivo is not without difficulties (see below).

\section{A new designer approach for targeting CTLA-4}

In this issue of the JCI, Fife et al. describe a novel approach for targeting CTLA-4 on activated $T$ cells in order to inhibit autoimmune responses in the NOD mouse (24). They accomplished this by selectively expressing a single-chain, membranebound anti-CTLA-4 antibody (scFv) on B cells. B cells from $\mathrm{scFv}$ transgenic mice not only inhibited $\mathrm{T}$ cell responses to antigen presented by these $B$ cells in vitro and in vivo but also conferred significant protection against disease in the NOD mouse model of autoimmune diabetes. The mechanism of regulation is thought to be the prevention of autoreactive $T$ cell expansion and development of effector function in the pancreatic lymph nodes. This system takes advantage of CTLA-4 engagement by transgenic $B$ cells in the lymph node, where CTLA-4 is thought to exert its effects on activated $T$ cells (25). The protection was seen even in a system relatively deficient of regulatory cells and in which there was no restoration of these cells upon expression of scFv on B cells of NOD.B7 DKO mice, indicating the independence of this mechanism from Tregs. On the other hand, the superior protection of standard NOD mice from diabetes compared with the Tregdeficient NOD.B7 DKO model may suggest that transgenic $B$ cells mediate some of the protective effect by engagement of CTLA-4 on Tregs and enhancement of their function. The incomplete protection from diabetes may be due to interaction of autoreactive $\mathrm{T}$ cells with other APCs. Nevertheless, this strategy presents a novel designer approach to specifically immunomodulating an autoimmune response. Whether this strategy will be successful in restoring tolerance to autoantigens after the establishment of overt autoimmunity remains to be seen.

\section{Challenges to targeting negative costimulatory pathways}

Location is important. Recently, novel CD28 family members, including ICOS, programmed death-1 (PD-1), and $\mathrm{B}$ and $\mathrm{T}$ lymphocyte attenuator (BTLA), and new B7 family members, including ICOS ligand (ICOSL) (also known as B7-H), PD-1 ligand (PD-L1) (also known as B7-H1), PD-L2 (B7-DC), B7-H3, and B7-H4, having varied expression patterns among both lymphoid and nonlymphoid (parenchymal) tissues, have been described, providing further insight into the complexity of the functions of $T$ cell costimulatory pathways $(26,27)$. Until recently, the regulation of the immune response by costimulatory molecules was thought to occur exclusively in the lymphoid organs at the time of the priming of the T cells by APCs and/or in the target organs by the interaction of infiltrating $\mathrm{T}$ cells with APCs. The expression of B7-1 and B7-2 is largely restricted to professional APCs while the novel B7 family members, including ICOS ligand, PD-L1, and PD-L2, have a much wider expression pattern, including parenchymal tissue of the islets, heart, brain, and placenta (28-30). We have recently demonstrated that tissue expression of the inhibitory molecule PD-L1 on islet cells mediates $\mathrm{T}$ cell tolerance by regulating the autoimmune response in the target tissue and at sites of inflammation (31).

Timing is important. Another complicating factor in targeting costimulatory pathways for the prevention or cure of autoimmune diseases is the differential response of the disease to the same intervention at different stages of disease. For example, in a NOD mouse model of autoimmune diabetes, CTLA-4 blockade precipitated disease only when administered prior to the onset of insulitis, indicating that this pathway may be operative only in the induction phase of the disease $(25,28)$. Conversely, one might speculate that, while strategies targeting the CTLA-4 pathway during the induction phase of the disease may be effective, later administration, especially after the establishment of overt diabetes, may not be able to restore $\mathrm{T}$ cell tolerance to the islet antigens. However, this requires further investigation since it is possible that CTLA-4 negative regulation may continue to be operative within the target organ through its function in Tregs. In contrast to CTLA-4, PD-1 pathway blockade precipitated diabetes when an anti-PD-L1 mAb was administered at any time point throughout the lifespan of the NOD mouse, indicating that this pathway is operative during induction and progression of the autoimmune response (28). Therefore, it is important to choose to target the appropriate pathway at the appropriate stage of the autoimmune response and disease process in order to obtain the desired optimal outcome. In this regard, studies to address interactions among the negative pathways, such as those involving CTLA-4:B7 versus PD-1:PDL1/2, vis-à-vis timing and locale of intervention will be valuable in the development of novel approaches to obtain optimal results from targeting negative costimulatory molecules.

\section{Potential novel approaches to harnessing negative regulatory pathways to prevent or cure autoimmune diseases}

First, an ideal therapeutic approach from the standpoint of ease of use in a clinical setting would be to develop biologicals (e.g., antibodies/fusion proteins) that deliver a negative signal via the various inhibitory costimulatory pathways (Figure 1). However, this approach has proven to be difficult, as researchers have not been able to develop a reliable signaling biological reagent, particularly for in vivo systems. $\mathrm{T}$ cell costimulatory molecules deliver either a positive or a negative signal only in conjunction with a TCR-mediated signal that is enhanced or dampened, respectively. Thus, CTLA-4 signaling requires crosslinking of CTLA- 4 with its ligands, B7-1 or B7-2, in conjunction with TCR engagement $(32,33)$. Accordingly, to be able to deliver the appropriate physiological signal by a biological, the agent must be able to cross-link its target receptor together with the TCR signal both temporally and spatially. Bluestone and colleagues have previously reported that coordinate ligation of CTLA-4, with cell surface-linked, single-chain anti-CTLA-4 antibody, and TCR on the same APC (in cis) is essential for signal attenuation (34). 


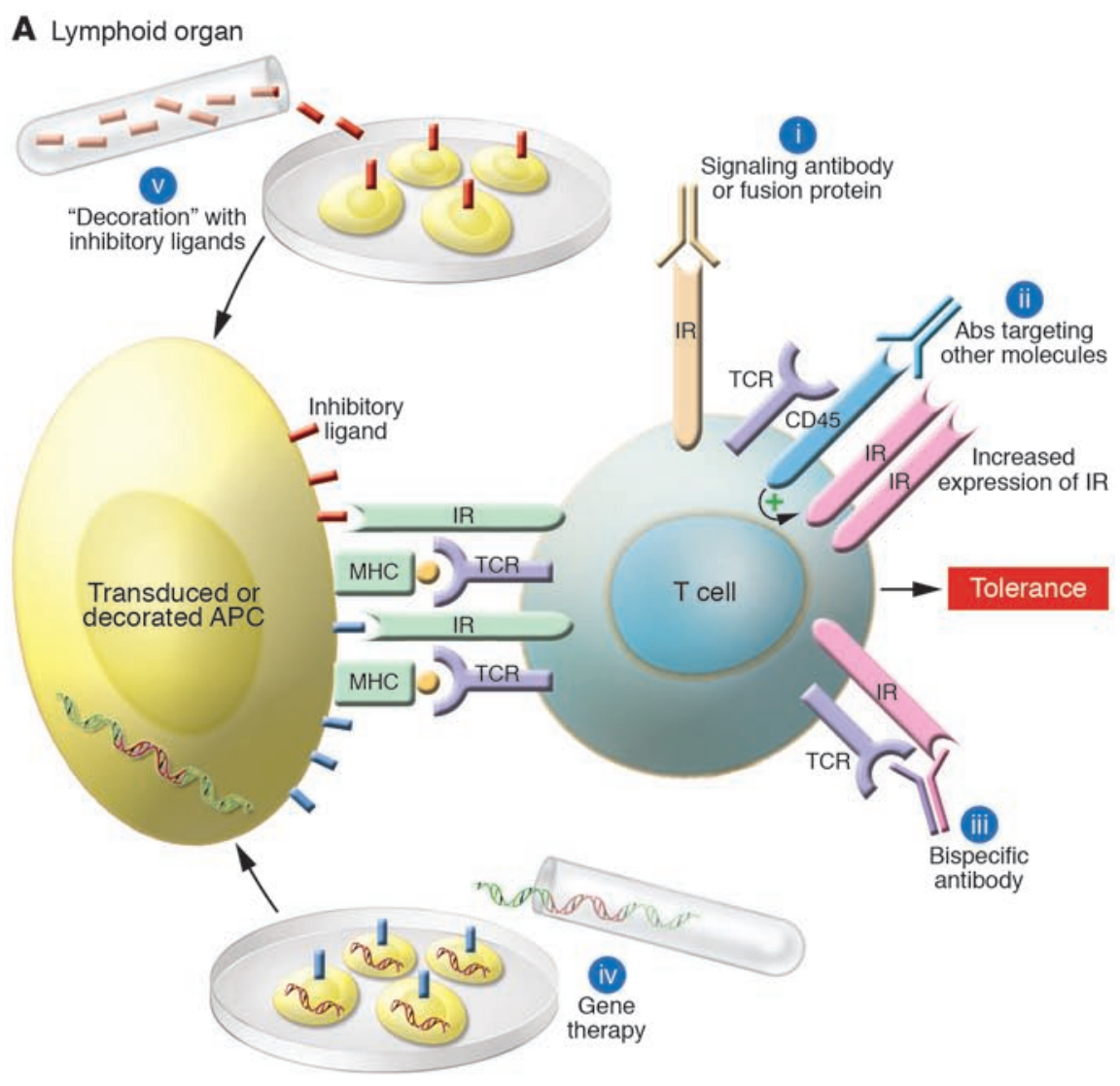

B Target organ

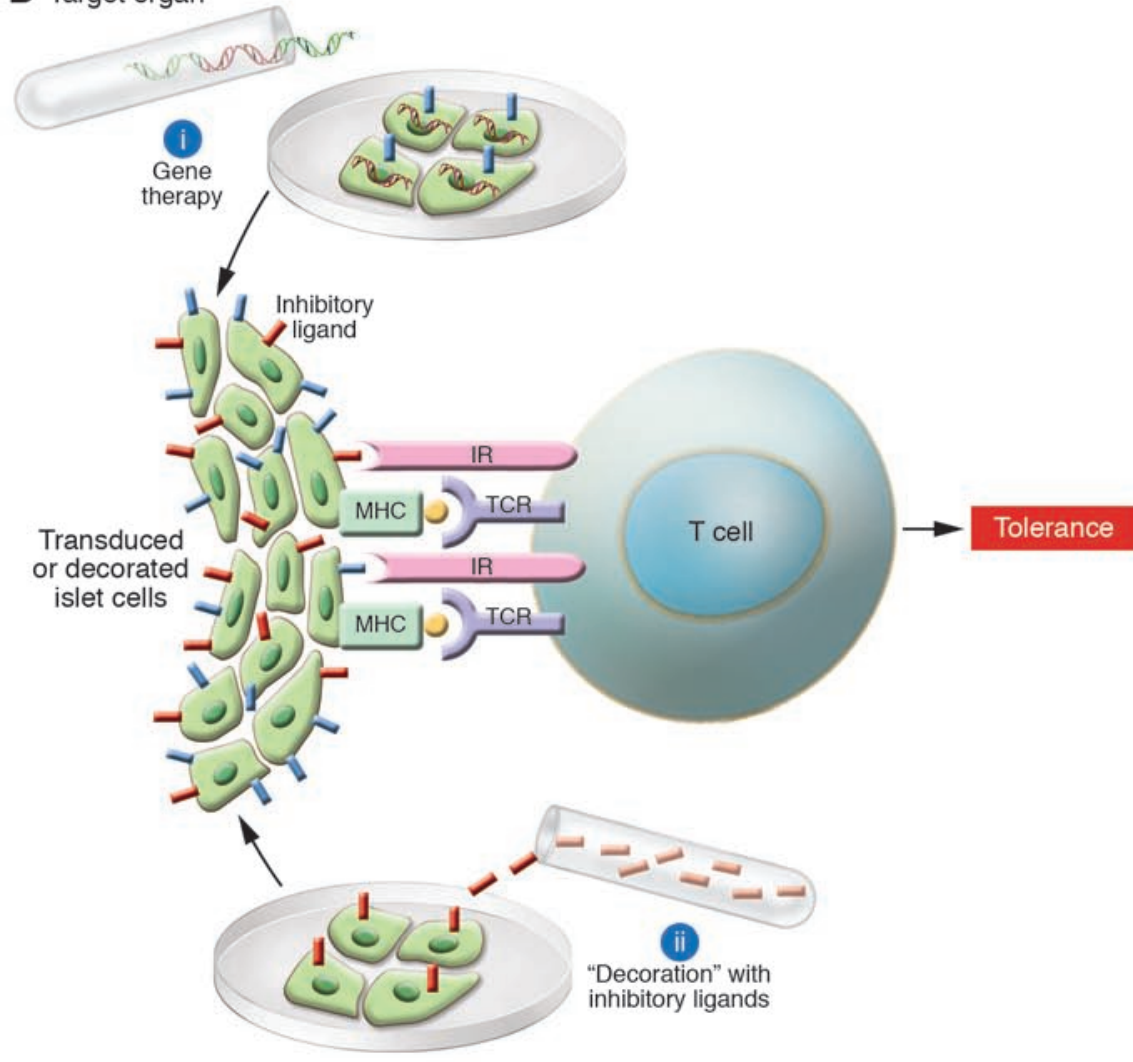

\section{Figure 1}

Schematic of potential approaches to harnessing negative regulatory pathways for the prevention or cure of autoimmune diseases. (A) Potential strategies operating within the lymphoid organs include the following: biologicals delivering a negative signal through the inhibitory receptors (IRs) on activated T cells (i); antibodies targeting other molecules (ii), which in turn enhance expression and function of negative costimulatory receptors on activated T cells; bispecific antibodies (iii) targeting TCR and inhibitory receptors simultaneously, thereby delivering a negative signal; gene therapy (iv) or "coating" (v) of APCs using ligands or antibodies targeting inhibitory receptors. (B) Potential strategies operating within the parenchyma of target organs include the following: gene therapy of target tissue (e.g., islets) and passenger APCs, with ligands or antibodies targeting inhibitory receptors (i); coating or "decoration" of target tissue (e.g., islets) and passenger APCs with ligands of inhibitory receptors (ii).

One potential approach would be to develop a bispecific antibody to simultaneously engage the relevant antigen and an inhibitory receptor (35). This approach has been demonstrated to be effective in an experimental mouse model of thyroiditis utilizing bispecific antibodies against thyroid-stimulating hormone and CTLA-4 (36). A major limitation of this strategy, however, is the need to identify immunodominant antigens for each disease and then generate disease-specific reagents targeting that particular antigen and an inhibitory receptor simultaneously. A second approach would be to target other molecules, such as CD45RB, which has been shown to promote tolerance by actually augmenting natural expression of inhibitory receptors (e.g., CTLA-4 in the case of anti-CD45RB) that in turn mediate tolerance in vivo (37-39).

A third approach is related to the discovery of a novel isoform of CTLA-4, ligand-independent CTLA-4 (liCTLA-4), which lacks the B7-binding domains. This isoform is highly expressed on memory/ regulatory $\mathrm{T}$ cells and has been described as inhibiting $\mathrm{T}$ cell responses. Recent evidence suggests that genetic variations of the expression of alternative spliced forms of CTLA-4 may contribute to susceptibility to autoimmunity (40). This discovery may provide the rationale to develop new immunomodulatory therapies for autoimmunity and other immune-mediated diseases. In addition, better understanding of 
the intracellular signaling pathways utilized by negative costimulatory molecules (ligand dependent or independent) may allow the development of small molecules that effectively target these pathways for therapeutic purposes.

A fourth approach would be to utilize gene therapy to target expression of the construct described by Fife et al. (24) or ligands of other inhibitory receptors, such as PD-1, on APCs, including macrophages, dendritic cells, and B cells. This approach could perhaps even be extended to parenchymal tissue, for example, the islets, in the context of islet transplantation for autoimmune diabetes. A fifth approach would be to "decorate" the membrane of APCs or graft tissue with inhibitory molecules utilizing a method for biotinylation of cell membranes and "coat" the membrane with chimeric proteins comprised of streptavidin and an inhibitory molecule. This seems to be a promising strategy, and as proof of principle, such an approach has been demonstrated to be effective in the prevention of islet allograft rejection by targeting the Fas/Fas ligand pathway (41).

\section{Conclusion}

Over the past 2 decades, the concept of $\mathrm{T}$ cell costimulation has evolved from the basic principle that $\mathrm{T}$ cells require a second costimulatory signal for full activation to the knowledge that costimulatory signals can both enhance (positive) and inhibit (negative) $\mathrm{T}$ cell activation and that some of the costimulatory receptor ligands are expressed not only on APCs but also on parenchymal cells that may be important in regulating immune responses in the target organ (26). As a result of these complexities, targeting negative costimulatory pathways to inhibit immune responses has proven to be tricky and challenging but at the same time has opened new opportunities for developing novel approaches of immunomodulation to prevent or cure autoimmune diseases.

\section{Acknowledgments}

M.J. Ansari is supported by an American Society of Transplantation-Juvenile Diabetes Research Foundation joint fellowship grant award. M.H. Sayegh is supported by the National Institute of Allergy and Infectious Diseases (grants P01 AI041521 and P01 AI056299) and a grant from the Juvenile Diabetes Research Foundation Center on Immunological Tolerance in Type I
Diabetes. We thank David M. Rothstein for critical review of this manuscript.

Address correspondence to: Mohamed H. Sayegh, Transplantation Research Center, 221 Longwood Avenue, Boston, Massachusetts 02115, USA. Phone: (617) 732-5259; Fax: (617) 732-5254; E-mail: msayegh@ rics.bwh.harvard.edu.

1. Bretscher, P., and Cohn, M. 1970. A theory of selfnonself discrimination. Science. 169:1042-1049.

2. Lafferty, K.J., and Woolnough, J. 1977. The origin and mechanism of the allograft reaction. Immunol. Rev. 35:231-262.

3. Lafferty, K.J., Prowse, S.J., Simeonovic, C.J., and Warren, H.S. 1983. Immunobiology of tissue transplantation: a return to the passenger leukocyte concept. Annu. Rev. Immunol. 1:143-173.

4. June, C.H., Ledbetter, J.A., Linsley, P.S., and Thompson, C.B. 1990. Role of the CD28 receptor in T-cell activation. Immunol. Today. 11:211-216.

5. Harding, F.A., McArthur, J.G., Gross, J.A., Raulet, D.H., and Allison, J.P. 1992. CD28-mediated signalling co-stimulates murine $\mathrm{T}$ cells and prevents induction of anergy in T-cell clones. Nature. 356:607-609

6. Walunas, T.L., et al. 1994. CTLA-4 can function as a negative regulator of $\mathrm{T}$ cell activation. Immunity. 1:405-413.

7. Krummel, M.F., and Allison, J.P. 1995. CD28 and CTLA-4 have opposing effects on the response of $\mathrm{T}$ cells to stimulation. J. Exp. Med. 182:459-465.

8. Eggena, M.P., et al. 2004. Cooperative roles of CTLA-4 and regulatory T cells in tolerance to an islet cell antigen. J. Exp. Med. 199:1725-1730.

9. Read, S., Malmstrom, V., and Powrie, F. 2000. Cytotoxic $\mathrm{T}$ lymphocyte-associated antigen 4 plays an essential role in the function of CD25(+)CD4(+) regulatory cells that control intestinal inflammation. J. Exp. Med. 192:295-302.

10. Takahashi, T., et al. 2000. Immunologic self-tolerance maintained by $\mathrm{CD} 25(+) \mathrm{CD} 4(+)$ regulatory $\mathrm{T}$ cells constitutively expressing cytotoxic $\mathrm{T}$ lymphocyte-associated antigen 4. J. Exp. Med. 192:303-310.

11. Bluestone, J.A., and Abbas, A.K. 2003. Natural versus adaptive regulatory T cells. Nat. Rev. Immunol. 3:253-257.

12. Tang, Q., et al. 2004. Distinct roles of CTLA-4 and TGF-beta in CD4+CD25+ regulatory $\mathrm{T}$ cell function. Eur. J. Immunol. 34:2996-3005.

13. Zheng, S.G., et al. 2006. TGF-beta requires CTLA-4 early after T cell activation to induce FoxP3 and generate adaptive CD4+CD25+ regulatory cells. J. Immunol. 176:3321-3329.

14. Salomon, B., et al. 2000. B7/CD28 costimulation is essential for the homeostasis of the CD4+CD25+ immunoregulatory $\mathrm{T}$ cells that control autoimmune diabetes. Immunity. 12:431-440.

15. Perez, V.L., et al. 1997. Induction of peripheral T cell tolerance in vivo requires CTLA-4 engagement. Immunity. 6:411-417.

16. Bluestone, J.A. 1997. Is CTLA-4 a master switch for peripheral $\mathrm{T}$ cell tolerance? J. Immunol. 158:1989-1993.

17. Markees, T.G., et al. 1998. Long-term survival of skin allografts induced by donor splenocytes and anti-CD154 antibody in thymectomized mice requires CD4(+) T cells, interferon- $\gamma$, and CTLA4. J. Clin. Invest. 101:2446-2455.

18. Judge, T.A., et al. 1999. The role of CD80, CD86, and CTLA4 in alloimmune responses and the induction of long-term allograft survival. J. Immunol. 162:1947-1951.

19. Greenwald, R.J., Boussiotis, V.A., Lorsbach, R.B., Abbas, A.K., and Sharpe, A.H. 2001. CTLA- 4 regulates induction of anergy in vivo. Immunity. 14:145-155.

20. Bluestone, J.A., St. Clair, E.W., and Turka, L.A. 2006. CTLA4Ig: bridging the basic immunology with clinical application. Immunity. 24:233-238.

21. Rothstein, D.M., and Sayegh, M.H. 2003. T-cell costimulatory pathways in allograft rejection and tolerance. Immunol. Rev. 196:85-108.

22. Genovese, M.C., et al. 2005. Abatacept for rheumatoid arthritis refractory to tumor necrosis factor alpha inhibition. N. Engl. J. Med. 353:1114-1123.

23. Vincenti, F., et al. 2005. Costimulation blockade with belatacept in renal transplantation. N. Engl. J. Med. 353:770-781.

24. Fife, B.T., Griffin, M.D., Abbas, A.K., Locksley, R.M., and Bluestone, J.A. 2006. Inhibition of T cell activation and autoimmune diabetes using a $\mathrm{B}$ cell surface-linked CTLA-4 agonist. J. Clin. Invest. 116:2252-2261. doi:10.1172/JCI27856.

25. Luhder, F., Hoglund, P., Allison, J.P., Benoist, C., and Mathis, D. 1998. Cytotoxic T lymphocyte-associated antigen 4 (CTLA-4) regulates the unfolding of autoimmune diabetes. J. Exp. Med. 187:427-432.

26. Khoury, S.J., and Sayegh, M.H. 2004. The roles of the new negative $T$ cell costimulatory pathways in regulating autoimmunity. Immunity. 20:529-538.

27. Greenwald, R.J., Freeman, G.J., and Sharpe, A.H. 2005. The B7 family revisited. Annu. Rev. Immunol. 23:515-548.

28. Ansari, M.J., et al. 2003. The programmed death-1 (PD-1) pathway regulates autoimmune diabetes in nonobese diabetic (NOD) mice. J. Exp. Med. 198:63-69.

29. Salama, A.D., et al. 2003. Critical role of the programmed death-1 (PD-1) pathway in regulation of experimental autoimmune encephalomyelitis. J. Exp. Med. 198:71-78.

30. Guleria, I., et al. 2005. A critical role for the programmed death ligand 1 in fetomaternal tolerance. J. Exp. Med. 202:231-237.

31. Keir, M.E., et al. 2006. Tissue expression of PD-L1 mediates peripheral T cell tolerance. J. Exp. Med. 203:883-895.

32. Egen, J.G., and Allison, J.P. 2002. Cytotoxic T lymphocyte antigen- 4 accumulation in the immunological synapse is regulated by TCR signal strength. Immunity. 16:23-35.

33. Chikuma, S., Imboden, J.B., and Bluestone, J.A. 2003. Negative regulation of $T$ cell receptor-lipid raft interaction by cytotoxic T lymphocyte-associated antigen 4. J. Exp. Med. 197:129-135.

34. Griffin, M.D., et al. 2000. Blockade of T cell activation using a surface-linked single-chain antibody to CTLA-4 (CD152). J. Immunol. 164:4433-4442.

35. Rao, S., et al. 2001. Targeted delivery of anti-CTLA-4 antibody downregulates $T$ cell function in vitro and in vivo. Clin. Immunol. 101:136-145.

36. Vasu, C., Gorla, S.R., Prabhakar, B.S., and Holterman, M.J. 2003. Targeted engagement of CTLA-4 prevents autoimmune thyroiditis. Int. Immunol. 15:641-654.

37. Fecteau, S., et al. 2001. CTLA-4 up-regulation plays a role in tolerance mediated by CD 45. Nat. Immunol. 2:58-63.

38. Ariyan, C., et al. 2003. Cutting edge: transplantation tolerance through enhanced CTLA-4 expression. J. Immunol. 171:5673-5677.

39. Salvalaggio, P.R., et al. 2006. Antigen exposure during enhanced CTLA-4 expression promotes allograft tolerance in vivo. J. Immunol. 176:2292-2298.

40. Vijayakrishnan, L., et al. 2004. An autoimmune disease-associated CTLA-4 splice variant lacking the $\mathrm{B} 7$ binding domain signals negatively in $\mathrm{T}$ cells. Immunity. 20:563-575.

41. Yolcu, E.S., Askenasy, N., Singh, N.P., Cherradi, S.E., and Shirwan, H. 2002. Cell membrane modification for rapid display of proteins as a novel means of immunomodulation: FasL-decorated cells prevent islet graft rejection. Immunity. 17:795-808. 